Article

\title{
Analysis of the Sieve Unit Inclination Angle in the Cleaning Process of Oat Grain in a Rotary Cleaning Device
}

\author{
Zbigniew Krzysiak $^{1}$, Waldemar Samociuk ${ }^{1, *}$, Janusz Zarajczyk ${ }^{2}$, Zdzisław Kaliniewicz ${ }^{3}$ (D), \\ Daniel Pieniak ${ }^{4}$ (D) and Marcin Bogucki ${ }^{5}$ \\ 1 Department of Mechanical Engineering and Automatics, University of Life Sciences in Lublin, 20-612 Lublin, \\ Poland; zbigniew.krzysiak@wp.pl \\ 2 Department of Horticulture Machinery and Tools, University of Life Sciences in Lublin, 20-612 Lublin, \\ Poland; janusz.zarajczyk@up.lublin.pl \\ 3 Faculty of Technical Sciences, Department of Heavy Duty Machines and Research Methodology, \\ University of Warmia and Mazury in Olsztyn, 10-719 Olsztyn, Poland; zdzislaw.kaliniewicz@uwm.edu.pl \\ 4 Department of Mechanics and Mechanical Engineering, Faculty of Transport and Computer Science, \\ University of Economics and Innovation, 20-209 Lublin, Poland; danielp60@o2.pl \\ 5 Department of Automation, Lublin University of Technology, 20-618 Lublin, Poland; mn.bogucki@gmail.com \\ * Correspondence: waldemar.samociuk@up.lublin.pl; Tel.: +48-81-531-97-55
}

Received: 29 January 2020; Accepted: 16 March 2020; Published: 18 March 2020

\begin{abstract}
The article presents some of the research on the wider work related to testing and improving a new rotary cleaning device. The new cereal grain cleaning and separation device can support the development of sustainable agriculture in terms of seed purity and quality. This device is especially useful for small and medium-sized farms. This device has the characteristics of a multi-stage screen aided by an air stream (i.e., by pneumoseparation). The prototype was constructed at the University of Life Sciences in Lublin. The research was conducted at a test stand, which allowed changes in the operating parameters of the cleaner and their measurement. The scope of the tests included determining the influence of the inclination angle of the sieve unit $(\alpha)$ on the effectiveness and efficiency of oat grain cleaning. The experiment was carried out using two rotational spindle speeds: 5 and $75 \mathrm{rpm}$. The quality of the separation and cleaning of oat grains was evaluated using six cleaning process parameters: plump grain mass separation coefficient $\left(S_{p g}\right)$, fine grain separation coefficient $\left(S_{f g}\right)$, fine impurities separation coefficient $\left(S_{f i}\right)$, chaff separation coefficient $\left(S_{c h}\right)$, total coefficient of cleaning effectiveness $(E)$, plump grain cleaning efficiency $\left(q_{p g}\right)$. Results showed the significant effect of the sieve drum inclination angle on the efficiency and effectiveness of oat grain cleaning.
\end{abstract}

Keywords: rotary cleaning device; grain separation; oat grain; conical sieve

\section{Introduction}

Agriculture and food production are essential elements of economy and society. Organic farming, which uses natural resources prudently, is essential for our food production and for our quality of life-today, tomorrow, and for future generations [1,2]. Organic farming is one of the most developed and accepted production systems from the aspect of environmental sustainability [3-5]. Realization of the sustainable agriculture concept requires the implementation of technological progress to reasonably intensify production and at the same time limit the degradation of soil production potential. It is necessary to expand and modernize the technical infrastructure of rural areas and the farms [6].

In particular, technological progress is needed in the area of agricultural machinery intended for small and medium-sized farms. Development requires the introduction of new machine and 
equipment implements to the market in every field. One of the most important processes for agriculture that requires improvement is grain cleaning and separation. Agricultural products, depending on the purpose, may need to be cleaned or sorted on suitable machines on the processing lines in order to prepare the raw material for processing [7]. Process of cleaning grain is aimed at separating impurities from the customs grain [8]. The requirements for cleanliness are different, depending on the level of qualification of standard grain. The highest demands are placed on consumer grains processed in the pharmaceutical and food industries. Process of cleaning is the separation of unnecessary or even harmful admixtures (impurities) from usable material [9]. Sorting is the second important process, especially in seed production. It is a division of usable material into groups (classes) of different utility value (usefulness). It consists of dividing the purified mass of grain into fractions differing in quality (mainly geometric parameters). The purity of the grain is determined by the percentage of impurities [10-12]. Cleaned grains and seeds significantly reduce the number of weeds in the field, which could be eliminated during the vegetation process to obtain the desired crop. This reduces the number of plant protection products needed to maintain plantations in good agricultural condition. The reduced consumption of chemicals in organic farming is the main criteria for which the consumers choose products [13]. Separation also improves the crop quality because quality grains and seeds can be sorted and others (split, small) eliminated. Quality grain should result in the growth of better, healthier plants which may reduce the need for use of artificial fertilizers. These possible benefits are in accordance with the sustainable use of soil resources and could positively affect the natural environment and development of society as a part of the ecosystem. Research on the cleaning process of grains and seeds may lead to a design of improved cleaning devices that sort grains to eliminate weed seeds.

Modern cleaning devices have high precision requirements for separation and cleaning, good efficiency, as well as the ability to adjust in a wide range of operating parameters and low noise $[14,15]$. It should be noted that many types of cleaning devices with different construction design are used [10,16]. Devices with rotating sieves have many advantages, such as no need for leveling in the transverse direction to the longitudinal axis, no distortion of the screen with excessive load, a larger usable screen surface (with the same dimensions of the device). In comparison with the classic grain and seed cleaners the authors' proposed new rotary cleaning device replaces the vibrating motion of the flat sieves with a rotary motion of the conical screen assembly. This has resulted in a significant reduction of noise emissions during the operation of the rotating cleaning device and increase in the durability of its individual components. Vibrations of machine elements, especially of large mass, are usually the cause of instability of machines, as well as may lead to their destruction $[10,16]$. However, the main idea was to shorten the grain cleaning process by eliminating the usage if a trieur (indented cylinder separator) to isolate the damaged grain, because this is done on a fine grain sieve. In addition, the cleaning process has been shortened due to the replacement of the initial, main, and final stages with one stage.

The new original part is a three-segment conical sieve through which individual fractions are separated. The separation process of the fourth fraction is pneumatically supported by a fan located at the end of the conical sieve drum housing. Air flows through the increasing cross-section of the sieve housing (diffuser). The use of two transfer blades located in the first segment (for separating small impurities) is a novelty. They cause the even distribution of the cleaned mixture and transfer it to the upper part of the sieve, from where the tested mass falls into the air stream generated by the exhaust fan [17]. Determining the relationship between the parameters of the new cleaning device may enable proper regulation and control of the cleaning process and may have a measurable effect on the product quality. The above positive features encouraged development of a prototype of a new rotary cleaning device and for research aimed at determining the range of its technological operating parameters to be undertaken. This may enable its implementation into production and usage in industry. The appropriate selection of parameter values requires experiments on the prototype of the device, according to a carefully prepared investigation scheme. The goal of the presented paper is to analyze the sieve unit (sieve drum) inclination angle in the rotary cleaning device and to determine the 
inclination value. This may increase performance of an optimized process of oat grain cleaning and removal of impurities contained in these grains. At this stage of the research we assumed constant moisture of the grain mixture, and a constant feeding capacity and fan speed.

Cleaning devices aimed at achieving higher cleaning efficiency and versatility to adapt them to various types of seeds currently play an important role $[10,18]$. However, this is not easy, because despite similar impurities, there are seeds with different properties. The problem of analyzing the cleaning process of various granular materials was the subject of some research [19-24]. The separation process depends on many factors including physical, geometric, and aerodynamic properties of the granular material $[25,26]$.

In the cleaning and segregation tests oat was used that gives an indispensable portion of energy to both humans and animals. Oats are characterized by a very high nutritional value due to two times more oil than other grains. These are mainly polyunsaturated fatty acids, which in addition to energy supply have a beneficial effect on health $[27,28]$. Oats are a uniquely nutritious food as they contain an excellent lipid profile and high amounts of soluble fiber [29,30]. It is necessary to know the physical characteristics of seeds that can form the basis for their separation and the processes governing them to create the most efficient cleaning devices [31]. An especially important influence on seed cleaning is the moisture of cleaned products [32-34].

\section{Materials and Methods}

\subsection{Investigated Device}

The investigated device is a rotary cleaning device prototype, patented in 2012 [17]. The separation of the cleaned mix, consisting of oat grains and impurities, is done on sifting sieves supported with an air stream to separate the chaff. The main idea of the applied cleaning method is to combine sifting with pneumatic cleaning $[17,35]$. The constructed device can be used as a stationary cleaner for cereal grains and seeds. The research was conducted at a test stand equipped with measurement devices for laboratory tests of cleaning and separation processes to assess performance of the investigated prototype (Figure 1). The design of the machine was developed and performed in accordance with safety standards, with an implemented safety function, developed on the basis of risk analysis [36].

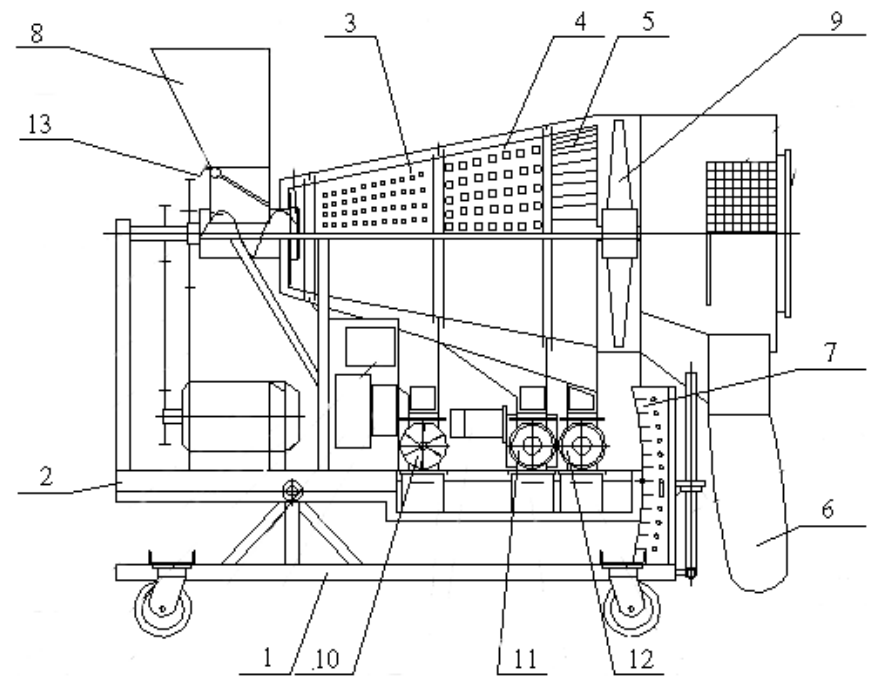

Figure 1. View of the stationary rotary cleaning device: 1-stationary frame, 2-mobile frame, 3-fine impurity segment, 4-fine grain segment, 5-plump grain segment, 6-chaff outlet container (bag), 7-indicator of sieve drum inclination angle, 8-hopper, 9-blower, 10-rotary valve of fine impurity segment, 11-rotary valve of fine grain, 12-rotary valve of plump grain segment, 13-valve closing mixture outlet. 
The new stationary cleaning device provides:

- The possibility of continuous speed regulation of the cone screen.

- The possibility of continuous fan speed control.

- The possibility of change of the power bandwidth.

- The possibility of chaff collecting.

- The possibility of selecting the cleaned mass in four fractions.

- The possibility of changing of the inclination angle of the sieve drum.

- The ability to exchange sieves.

The characteristics of the technical parameters are shown in Table 1.

Table 1. Characteristics of technical and working conditions.

\begin{tabular}{cc}
\hline Parameter & Value \\
\hline Feed system for cleaned material & \\
\hline Container capacity $(\mathrm{kg})$ & 30 \\
\hline Required capacity $\left(\mathrm{kg} \cdot \mathrm{s}^{-1}\right)$ & 1.25 \\
\hline Size of container outlet gap for capacity of $1.25 \mathrm{~kg} \cdot \mathrm{s}^{-1}(\mathrm{~mm})$ & 2.6 \\
\hline Sieve drum & $0-240$ \\
\hline Drum angular velocity $(\mathrm{rpm})$ & $0-80$ \\
\hline Sieve opening size & 2.5 \\
\hline Fine impurity sieve $(\mathrm{mm})$ & 4 \\
\hline Fine grain sieve $(\mathrm{mm})$ & $-7.5-10.0$ \\
\hline Sieve drum inclination angle $\mathrm{K}\left({ }^{\circ}\right)$ & $0-720$ \\
\hline Blower angular velocity $(\mathrm{rpm})$ & \\
\hline
\end{tabular}

\subsection{Research Methodology}

For the purpose of this research, a special grain mix was prepared, containing oat grains and impurities. The main test material was oat grains gleaned with a combine harvester. The mix for analysis and assessment of the cleaning process consisted of four fractions in the following weight proportions: oat plump grains $(6.8 \mathrm{~kg})$, oat fine grains $(2.6 \mathrm{~kg})$, and fine impurities-poppy seeds $(0.2 \mathrm{~kg})$ and chaff $(0.4 \mathrm{~kg})$. Altogether, the prepared test mix weighed $10 \mathrm{~kg}$. The characteristics of the oat grain are shown in Table 2.

Table 2. Selected properties of oats (type Kasztan) and chaff used in experiments.

\begin{tabular}{ccc}
\hline & Property & Value \\
\hline Oat & Stalk length $(\mathrm{m})$ & 0.90 \\
\hline Spike length $(\mathrm{m})$ & $0.10-0.20$ \\
\hline Number of grains per spike & $20-45$ \\
\hline Grain mass/straw ratio & $0.3-0.7$ \\
\hline Grain yield $\left(\mathrm{t} \mathrm{ha}^{-1}\right)$ & 5.0 \\
\hline
\end{tabular}


Table 2. Cont.

\begin{tabular}{ccc}
\hline & \multicolumn{2}{c}{ Value } \\
\hline & Plump Grain & Fine Grain \\
\hline Grain length $(\mathrm{mm})$ & 10.203 & 8.70 \\
\hline Grain width $(\mathrm{mm})$ & 2.47 & 2.07 \\
\hline Grain thickness $(\mathrm{mm})$ & 3.09 & 2.95 \\
\hline 1000-grain weight $(\mathrm{g})$ & $18.5-30.25$ \\
\hline Grain moisture (\% by weight) & 11.0 & $15.5-23.8$ \\
\hline Moisture content $(\%)$ & \multicolumn{3}{c}{$0.006-0.008$} \\
\hline Length $(\mathrm{m})$ & 0.004 \\
\hline Width $(\mathrm{m})$ & \multicolumn{3}{c}{0.0001} \\
\hline Thickness $(\mathrm{m})$ &
\end{tabular}

The composition of the mixture was selected in such a way that it was similar to the cleaned material from a combine-harvester after harvest. However, due to the small mass of the mixture sample, the mass of impurities was increased to allow a better evaluation of the cleaning process. Oat grain came from the experimental farm of the University of Life Sciences in Lublin (Lublin, Poland). Fine impurities, i.e., weed seeds and sand occurring at harvest, were replaced by poppy seeds, which had similar physical and geometrical parameters (Table 3).

Table 3. Selected properties of Przemko cultivar of poppy and of sand.

\begin{tabular}{cc}
\hline & Mean Value \\
\hline Grain length $(\mathrm{mm})$ & $1.0-7.5$ \\
\hline Grain width $(\mathrm{mm})$ & $0.5-0.75$ \\
\hline Grain moisture (\% by weight) & 9.00 \\
\hline Morphine alkaloid content $(\%)$ & 0.05 \\
\hline Fat content $(\%)$ & 48 \\
\hline
\end{tabular}

The moisture of each component was as follows for plump grains and fine grains- $11 \%$, for fine impurities-9\%, and for chaff-9.3\%. After cleaning, each fraction was weighed using an electronic scale, exactly to $0.002 \mathrm{~kg}$. The time for the separation of each fraction was measured with a stopwatch. On the basis of the measured values we established, we calculated separation coefficients for each fraction and the total coefficient of cleaning effectiveness. The research was performed with the test stand of the cleaning device after selecting the sieve unit inclination angle $\alpha$, for instance, $\alpha=2.5^{\circ}$, and then fixing it with bolts. Next, the sample of the test mix was poured into the hopper. After activating the device, a constant rotational speed of the fan was established at 500 revolutions per minute (rpm) using a power inverter. Next, the value of the sieve drum rotational speed was selected in the range from 0 to $80 \mathrm{rpm}$, for instance, $5 \mathrm{rpm}$ with a 5-rpm step. Afterwards, the mixture outlet closing valve was opened, and the cleaned mix was fed into the sieve drum via gravitation supported by the feed screw. After the separation of fractions from the tested sample was completed and the fractions were separated into individual containers, each separated mix component was weighed on the electronic scale. During experiments, fine grain and plump grain separation times were measured with a stopwatch. Response surface methodology (RSM) was used to plan experiment [37]. Cereal grain cleaning is a nonlinear process that requires advanced control algorithms [38], therefore a nonlinear controller was connected to the system [39]. The measurements were performed for five angles, with five repetitions for the entire range of the sieve drum rotational speed. 


\subsection{Evaluating of Cleaning Effectiveness and Statistical Analysis}

To assess the separation process there were proposed mass separation coefficients for each fraction. To determine the separation performance the separation coefficient $S$ was introduced, defined by the formula below:

$$
S_{i}=\frac{m_{i}}{M_{i}}
$$

where: $m_{i}$-mass of substance separated after cleaning, $M_{i}$-precleaning mass of substance in the mixture, index $i=f i, f g, p g$, $c h$, the indices corresponding to each fraction: $f i-$ fine impurities, $f g$ - fine grain, $p g$-plump grain, ch—chaff.

The analysis of separation performance was based on the calculated total cleaning effectiveness coefficient $(E)[10]$ :

$$
E=\frac{m_{b}}{M_{b}}-\frac{m_{a}}{M_{p g}}
$$

where: $m_{a}$-plump grain mass in waste, $m_{b}$-impurity mass in waste, $M_{b}$-impurity mass in mixture, and $M_{p g}$-plump grain mass in mixture.

The plump grain mass in the waste, $m_{a}$, was defined as follows:

$$
m_{a}=M_{p g}-m_{p g}
$$

where: $M_{p g}$ —plump grain mass in mixture, $m_{p g}$ —plump grain mass after the cleaning process.

The mass of impurity in the waste $\left(m_{b}\right)$ was calculated using the following formula:

$$
m=m_{f i}+m_{f g}+m_{c h}
$$

where: $m_{f i}-$ fine impurity mass in the waste after cleaning, $m_{f g}$ - plump grain mass in the waste after cleaning, and $m_{c h}$-mass of chaff in the waste after cleaning.

The impurity mass in the mixture $M_{b}$ was defined as follows:

$$
M=M_{f i}+M_{f g}+M_{c h}
$$

where: $M_{f i}$-fine impurity mass in the mixture, $M_{f g}$-fine grain mass in the mixture, $M_{c h}$-chaff mass in the mixture.

The cleaning efficiency for plump grain $q_{p g}$ was defined as follows:

$$
q_{p g}=\frac{m_{p g}}{t_{p g}}
$$

where: $m_{p g}$-plump grain mass after the cleaning process, $t_{p g}$-time of separation process.

Statistical analysis was performed using Statistica (StatSoft, Inc., Tulsa, OK, USA). The effect of inclination angle was tested by one-way analysis of variance (ANOVA) at the level of significance of $p=0.05$, followed by a post-hoc Tukey's honest significance difference (HSD) test.

\subsection{Method for Measuring the Inclination Angle of the Sieve Unit}

The rotary cleaning device has a stationary frame and a mobile frame. The mobile frame is used as a structure to which the elements of the test stand are attached. In the front part, the stationary frame is connected to the mobile frame through a hinge, and the control mechanism (screw) of the sieve unit inclination angle is located in the back part (Figure 2). The control range of this mechanism is within $10^{\circ}$ upwards and $7.5^{\circ}$ downwards with respect to the horizontal position $\left(0^{\circ}\right)$. The reading of the inclination angle is done using the angular indicator located on the frame. Two flats are used for placing the mobile frame in a working position. These flats have holes located in $2.5^{\circ}$ steps and two bolts for locking the frame in the required position. The complete methodology of this research was elaborated according to the published guidelines, and it has been presented in our earlier 
papers $[40,41]$. The influence of the sieve drum inclination angle on the process of separating particular fractions was investigated at the blower rotational speed $v_{d}=500 \mathrm{rpm}$ and at two sieve drum rotational speed $v_{d}$ values, $v_{d}=5 \mathrm{rpm}$ and $v_{d}=75 \mathrm{rpm}$. Since this research was very labor-intensive, only two extreme values of the sieve drum rotational speed values were chosen for analysis. In this study, we assumed the following nomenclature: selecting the negative sieve drum angle decreases the sieve drum inclination angle, and the positive one increases it. Five values of the inclination angle $\alpha$ were analyzed, from $\alpha=-2.5^{\circ}$ through $\alpha=0^{\circ}$ to $\alpha=2.5^{\circ}$. These values were chosen on the basis of the analysis of earlier investigations of the combine harvester sieve unit, performed for the full range of the sieve drum inclination angle. No investigations were performed in this paper for inclination angle values above $2.5^{\circ}$ or below $-2.5^{\circ}$, as during the initial tests we observed that the cleaning process did not proceed properly, and the device did not fulfill its purpose. The preliminary investigations for other inclination angles yielded parabolic curves representing graphs of the analysis results. Therefore, the results were approximated using second order polynomials. The analysis of the inclination angle was limited to two characteristic sieve drum rotational speed values, the lower limit $v_{d}=5 \mathrm{rpm}$ and the upper limit $v_{d}=75 \mathrm{rpm}$ of the device working range. Repeated experiments according to the same program allow more information to be obtained about the research subject. Each experiment was repeated five times, as repetitions increased the precision of the measurements, and the influence of outliers on the final measurement could be decreased. Additionally, more information about the measured object was obtained, and the results better approached improved values in terms of optimization.

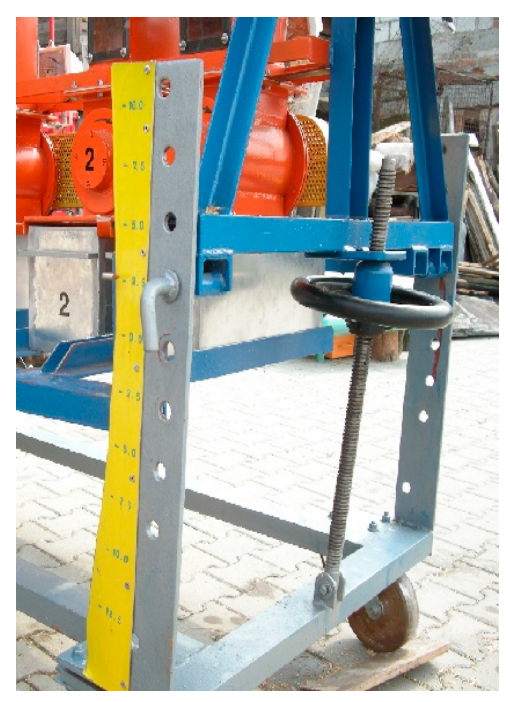

Figure 2. Indicator and control mechanism of the sieve unit inclination angle.

The constructed prototype of the cleaning device should be additionally equipped with a set of sieves for the fine impurities section and the fine grain section with various mesh sizes and spacing. Currently in the examined prototype of the equipment one mesh spacing pattern was used, the mesh size being chosen in such a way as to make it possible to examine the basic types of grain without the necessity to replace them and without the need to determine whether it affects the cleaning process in a significant way.

The mesh size was considerably larger than necessary to allow for free passage of individual seeds and grains of the separated fractions. Thanks to that it was possible to control the separation process so that more or less grain of plump grain would reach the fine grain fraction. Under dynamic operating conditions of the cleaning device the sieve mesh size did not matter for the lower range of the rotary speed of the sieve drum (3-10 rpm). Regarding the upper range of the rotary speed values of the sieve drum (60-75 rpm) the mesh size is extremely important in particular for the analyzed case, fine grain is considered the waste because as a result of the operation of the centrifugal force the plump grain gets to the fine grain fraction, therefore causing a disruption in the separation process and in the proper 
operation of the equipment. However, it does not matter when the fine grain and the plump grain fraction are one product (separation into three fractions). Such a selection of mesh size shows some significant principles of the device universality. The division into four fractions should be applied when it is necessary to separate fine grain and damaged grain that is considered a waste e.g., in seed production. In such a case the upper value (e.g., $70 \mathrm{rpm}$ ) of the recommended range of the rotary speed values of the sieve drum should be used. Regarding the needs of processing in the food industry, lower values (e.g., $45 \mathrm{rpm}$ ) of the recommended range of the rotary speed values of the sieve drum can be used because fine grain can be the product to be used for further processing e.g., for flour or fodder.

To sum up the conducted laboratory testing as well as the analysis of the results presented in the form of diagrams are shown above and in other works by the author [40]. It was found that the cleaning device is effective at cleaning of the oat grain, however, it requires further research under industrial conditions [41].

\section{Results and Discussion}

\subsection{Influence of the Sieve Drum Inclination Angle on the Cleaning Process}

In this section we analyze the influence of the sieve drum inclination angle on separation coefficients for each fraction, as well as the total coefficient of cleaning effectiveness and cleaning efficiency for the blower rotational speed $v_{b}=500 \mathrm{rpm}$ and feeding throughput $t_{f}=1.25 \mathrm{~kg} \cdot \mathrm{s}^{-1}$. Our goal was to separate all plump grains from the other ingredients of the mixture, and to achieve both maximal purity and maximal efficiency at the same time. These qualities are interrelated, so we expected tradeoffs while optimizing their values. All analyses were performed for two sieve drum rotational speed values, the lower limit $v_{d}=5 \mathrm{rpm}$ and the upper limit $v_{d}=75 \mathrm{rpm}$ of the drum rotation range. The diagrams illustrating the results of these analyses are included in the following subsections.

\subsection{Analysis of the Influence of Sieve Drum Inclination Angle on the Cleaning Process at $v_{d}=5 \mathrm{rpm}$}

The relationship between the plump grain separation coefficient $S_{p g}$ and the sieve drum inclination angle $\alpha$ is shown in Figure 3a. As we can see, the value of the oat plump grain separation coefficient $S_{p g}$ increased with the increase of the sieve drum inclination angle to about 1.384, where value of inclination angle is $\alpha=2.5^{\circ}$.

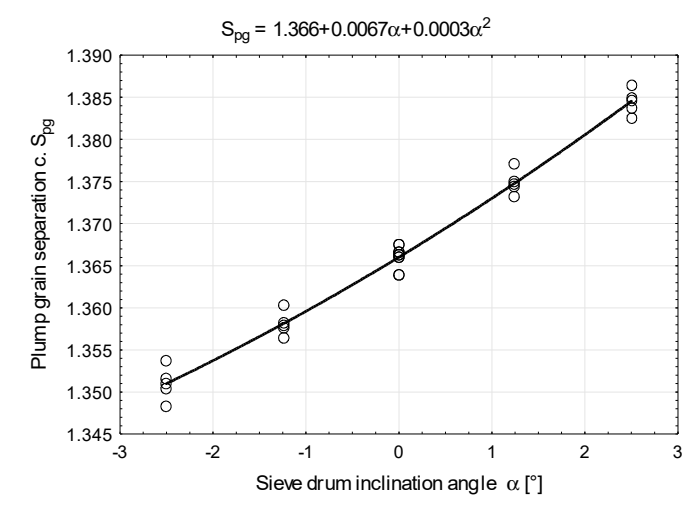

(a)

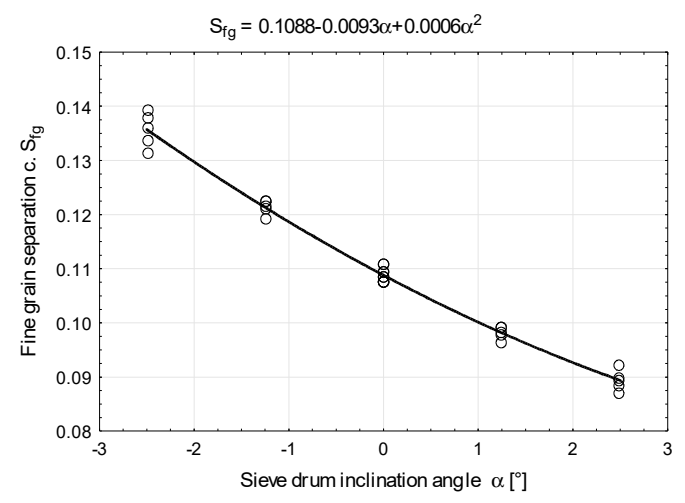

(b)

Figure 3. Cont. 


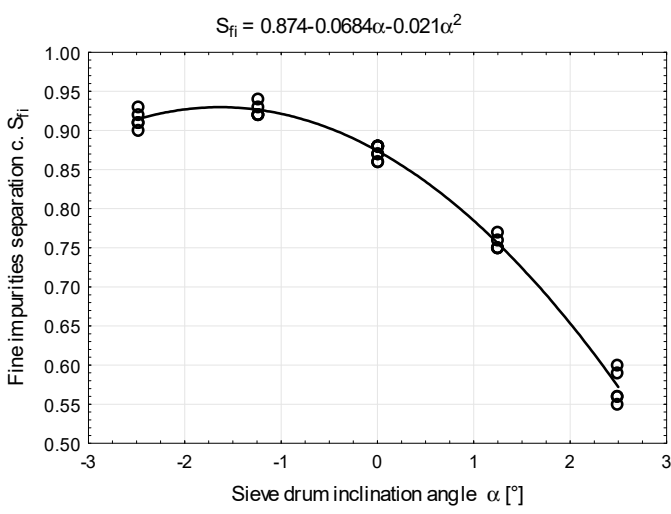

(c)

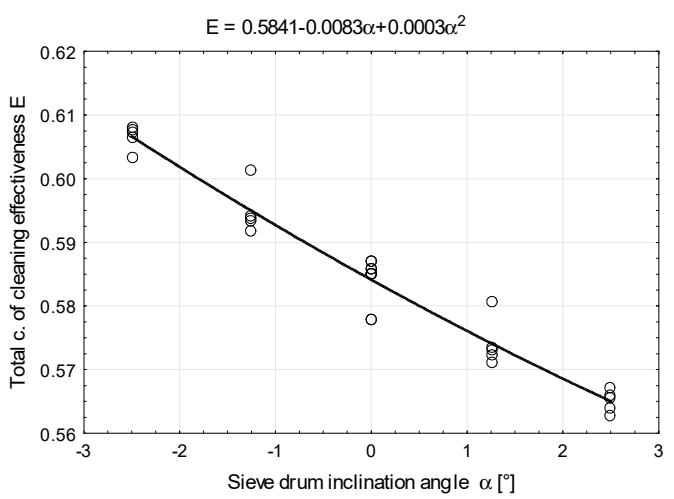

(e)

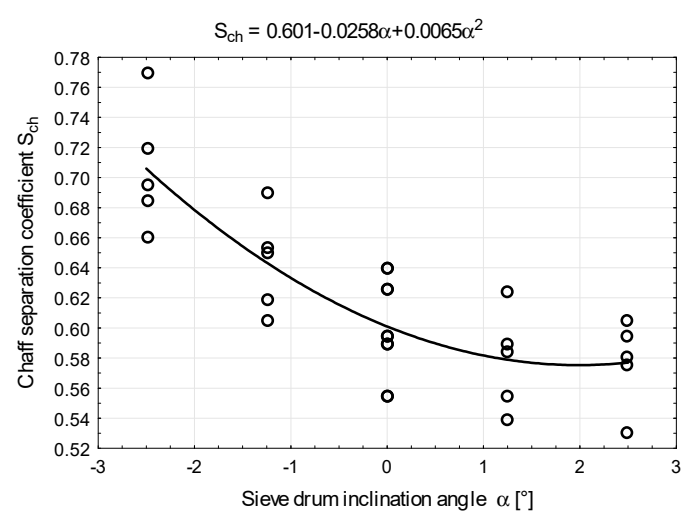

(d)

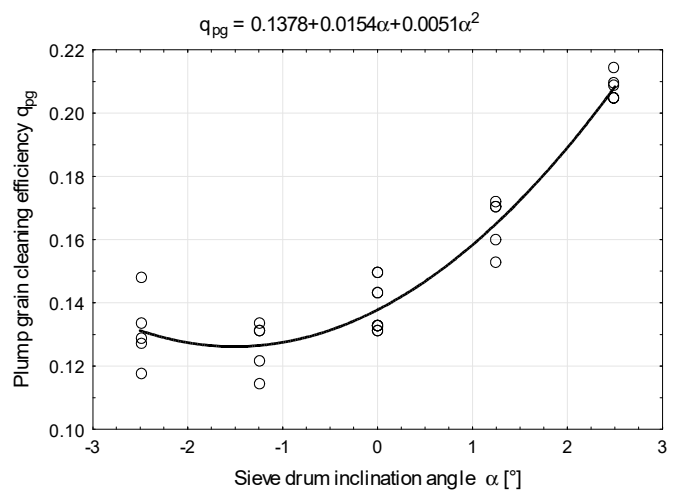

(f)

Figure 3. Analysis of the influence of the sieve drum inclination angle on the cleaning process for $v_{d}=5 \mathrm{rpm}$ : (a) plump grain separation coefficient $S_{p g}$, (b) fine grain separation coefficient $S_{f g}$, (c) fine impurities separation coefficient $S_{f i}$, (d) chaff separation coefficient $S_{c h}$, (e) total coefficient of cleaning effectiveness $E$, (f) plump grain cleaning efficiency $q_{p g}$.

The increase in the plump grain separation coefficient value $S_{p g}$ above unity is evidence of fine grains penetrating into the plump grain fraction. Fine grains were penetrating partially into plump grains within the entire range of the investigated angle, but the largest amount was penetrating at the $\alpha=2.5^{\circ}$ inclination angle. Therefore, this value of the inclination angle of the sieve drum is not correct.

The correlation graph of the oat fine grain separation coefficient $S_{f g}$ on the sieve drum inclination angle is shown in Figure $3 \mathrm{~b}$. The value of the fine oat grain separation coefficient has the highest value of 0.135 for the sieve drum inclination angle $\alpha=-2.5^{\circ}$. Then the value of $t$ decreases with the increase of the inclination angle of the sieve drum.

The graph of the fine grain separation coefficient is inverted compared to the plump grain separation coefficient (Figure 3a,b). Therefore, these coefficients are clearly interdependent. The largest amount of fine grains is released at the angle of inclination $\alpha=-2.5^{\circ}$, in this case the coefficient of fine grain separation $S_{f g}=0.135$. The correlation of the fine impurities separation coefficient $S_{f i}$ on the sieve drum inclination angle is presented in Figure 3c.

As can be seen, the course of the graph of the fine impurities separation coefficient has a local maximum $\left(S_{f i}=0.946\right)$-for the angle of inclination of about $\alpha=-1.75^{\circ}$. The graph of the correlation between the chaff separation coefficient $S_{c h}$ and the inclination angle of the sieve drum is shown in Figure $3 \mathrm{~d}$. This graph shows that the highest value of the chaff separation coefficient $S_{c h}=0.70$ was obtained at angle $\alpha=-2.5^{\circ}$.

The graph of the correlation between the total cleaning effectiveness coefficient $\mathrm{E}$ and the inclination angle of the sieve drum is shown in Figure 3e. As can be seen, the value of the total cleaning effectiveness coefficient $\mathrm{E}$ decreased with the increase of the inclination angle of the sieve drum. This change is caused by a change in the level of distribution of plump grains from fine grains, which, in this case, is 
treated as an impurity. In this case, the separation of plump grains from fine grain is disturbed while maintaining relative purity.

The highest cleaning effectiveness $E=0.606$ was obtained for angle $-2.5^{\circ}$. Cleaning effectiveness strictly depends on cleaning efficiency. While the total cleaning effectiveness coefficient is highest for inclination angle of sieve drum $\alpha=-2.5^{\circ}$, however, cleaning efficiency is much smaller than for angle $\alpha=2.5^{\circ}$. The highest efficiency $q_{p g}=0.22 \mathrm{~kg} / \mathrm{s}$ was obtained for angle $\alpha=2.5^{\circ}$. Cleaning efficiency course has local minimum $\left(q_{p g}=0.128 \mathrm{~kg} / \mathrm{s}\right)$ for inclination angle $\alpha=-1.75^{\circ}$.

The performed analysis of variance (one-way ANOVA) showed (Table 4) that for $v_{d}=5 \mathrm{rpm}$ the value of the sieve drum inclination angle had a significant effect on the obtained parameters (at a significance level of $p=0.05$ ). According to the post-hoc test (Tukey's test) the analyzed parameters differed significantly with two exceptions, i.e., for the plump grain separation coefficient $\left(S_{p g}\right)$ values obtained with $a-2.5^{\circ}$ and $1.25^{\circ}(1-2)$ sieve drum inclination angle, for groups $2-3,3-4,3-5$, and $4-5$ for $S_{c h}$ and for the $q_{p g}$ values for 1-2,1-3, and 2-3 groups (Table 5).

Table 4. Significance level for the ANOVA analysis.

\begin{tabular}{ccccccc}
\hline$v_{\boldsymbol{d}}(\mathbf{r p m})$ & $S_{p g}$ & $S_{f g}$ & $S_{f i}$ & $S_{c h}$ & $E$ & $\boldsymbol{q}_{p g}$ \\
\hline 5 & 0.00 & 0.00 & 0.00 & 0.00 & 0.00 & 0.00 \\
\hline
\end{tabular}

Table 5. Tukey's honest significance difference (HSD) test for the ANOVA.

\begin{tabular}{ccccccc}
\hline $\boldsymbol{v}_{\boldsymbol{d}}(\mathbf{r p m})$ & $S_{p g}$ & $S_{f g}$ & $S_{f i}$ & $S_{c h}$ & $E$ & $\boldsymbol{q}_{p g}$ \\
\hline $1-2$ & $\mathbf{0 . 4 7 9}$ & 0.000 & 0.000 & 0.047 & 0.000 & $\mathbf{0 . 8 8 5}$ \\
\hline $1-3$ & 0.000 & 0.000 & 0.000 & 0.000 & 0.000 & $\mathbf{0 . 5 7 5}$ \\
\hline $1-4$ & 0.000 & 0.000 & 0.000 & 0.000 & 0.000 & 0.000 \\
\hline $1-5$ & 0.000 & 0.000 & 0.000 & 0.000 & 0.000 & 0.000 \\
\hline $2-3$ & 0.000 & 0.000 & 0.000 & $\mathbf{0 . 1 7 1}$ & 0.000 & $\mathbf{0 . 1 0 9}$ \\
\hline $2-4$ & 0.000 & 0.000 & 0.000 & 0.038 & 0.000 & 0.000 \\
\hline $2-5$ & 0.000 & 0.000 & 0.000 & 0.031 & 0.000 & 0.000 \\
\hline $3-4$ & 0.000 & 0.000 & 0.000 & $\mathbf{0 . 7 4 6}$ & 0.000 & 0.000 \\
\hline $3-5$ & 0.000 & 0.000 & 0.000 & $\mathbf{0 . 6 8 5}$ & 0.000 & 0.000 \\
\hline $4-5$ & 0.000 & 0.000 & 0.000 & $\mathbf{1 . 0 0 0}$ & 0.001 & 0.000 \\
\hline
\end{tabular}

1-2 indicates the significance level between group 1 and group 2, 1-3 indicates the significance level between group 1 and group 3, etc. For group $1 \alpha=-2.5^{\circ}$, for group $2 \alpha=-1.25^{\circ}$, for group $3 \alpha=0^{\circ}$, for group $4 \alpha=1.25^{\circ}$, for group $5 \alpha=2.5^{\circ}$. Means that lack significant differences are shown in bold.

\subsection{The Analysis of the Influence of the Sieve Drum Inclination Angle on the Cleaning Process for $v_{d}=75 \mathrm{rpm}$}

The graph shown in Figure 4a presents the relation of the oat plump grain separation coefficient $S_{p g}$ on the sieve drum inclination angle. As can be seen, the graph course has a local minimum $\left(S_{p g}=1.05\right)$ for inclination angle circa $\alpha=-0.5^{\circ}$. This graph differs from the curve obtained for the plump grain separation coefficient for the sieve drum rotational speed $v_{d}=5 \mathrm{rpm}$ (Figure 3a). When $v_{d}=75 \mathrm{rpm}$, the coefficient $S_{p g}$ exceeds, only slightly, the unity value (about 1.12 for $\alpha=2.5^{\circ}$ ), whereas for $v_{d}=5 \mathrm{rpm}$ the value of $S_{p g}$ was much larger above unity for the entire investigated range of the sieve drum inclination angle $\alpha$. 


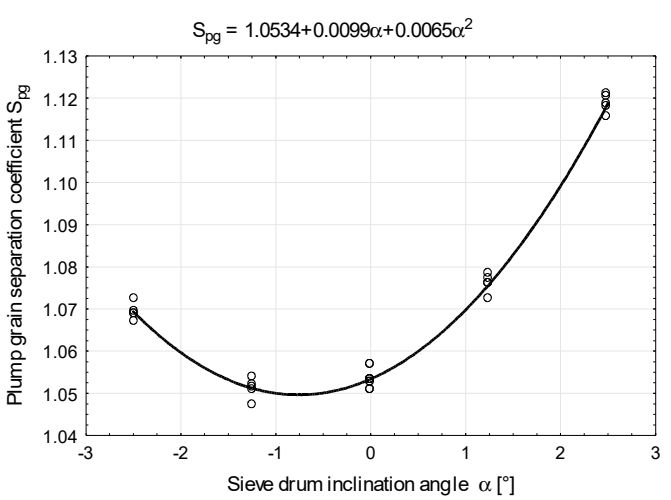

(a)

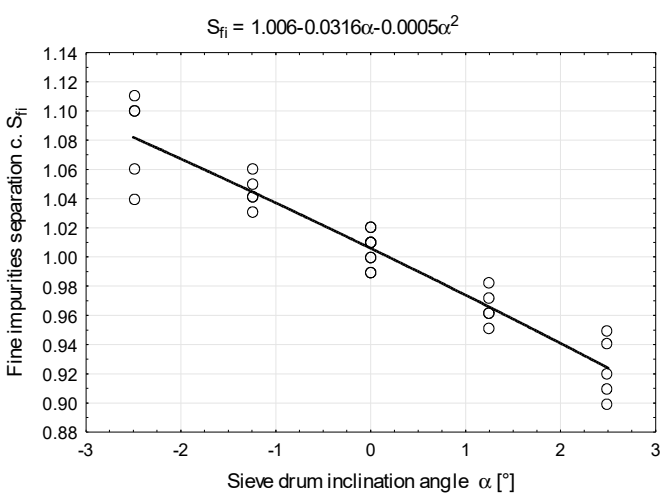

(c)

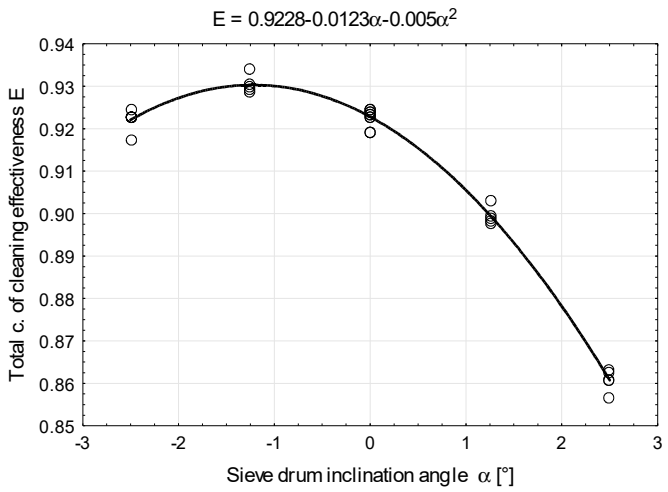

(e)

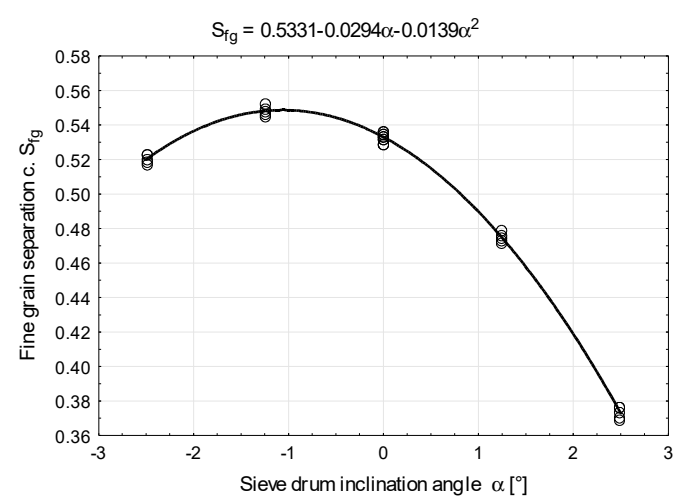

(b)

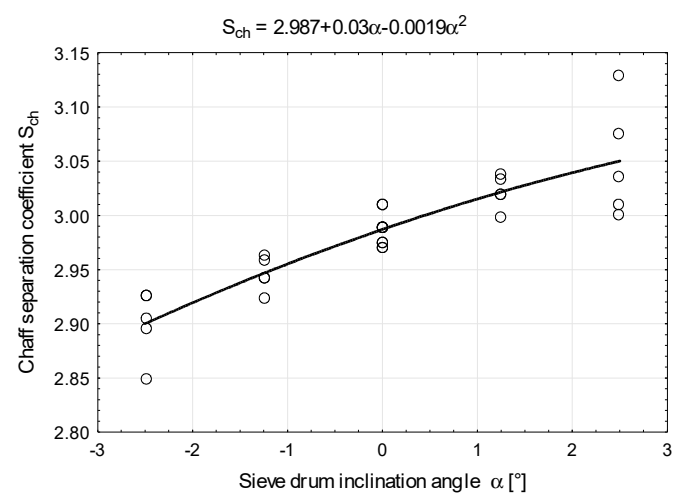

(d)

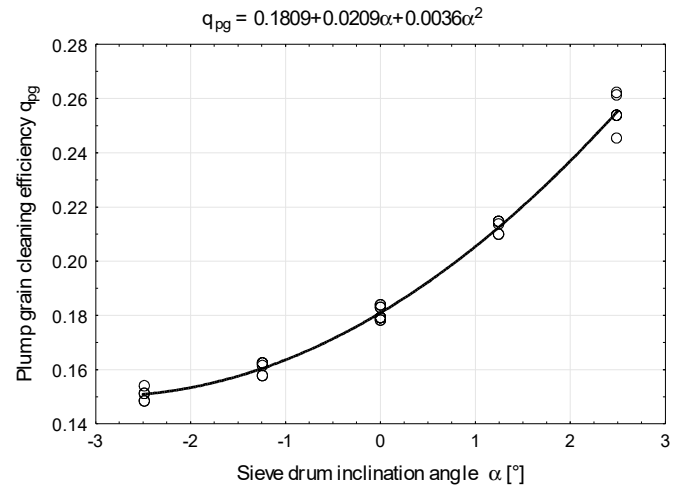

(f)

Figure 4. Analysis of the influence of the sieve drum inclination angle on the cleaning process for $v_{d}=75 \mathrm{rpm}$ : (a) plump grain separation coefficient $S_{p g}$, (b) fine grain separation coefficient $S_{f g}$, (c) fine impurities separation coefficient $S_{f i}$, (d) chaff separation coefficient $S_{c h}$, (e) total coefficient of cleaning effectiveness $E$, (f) plump grain cleaning efficiency $q_{p g}$.

The relation of the fine grain separation coefficient $S_{f g}$ on the sieve drum inclination angle is presented in Figure $4 \mathrm{~b}$. The fine grain separation coefficient $S_{f g}$ reached the highest value 0.55 when the inclination was about $-1^{\circ}$ and then decreased with the increase in the sieve drum inclination angle. This effect is mainly caused by the reduction of time of sifting the mixture in the sieve drum. The diagram of the correlation between the fine impurities separation coefficient $S_{f i}$ and the sieve drum inclination angle is shown in Figure 4c. As can be observed, the value of the fine impurities separation coefficient $S_{f i}$ decreased with the increase in the sieve drum inclination angle from $-2.5^{\circ}$ to $2.5^{\circ}$. The goal was to obtain values as close to unity as possible, so we concluded that the angle just above $0^{\circ}$ is recommended. This is caused by fine grains penetrating to fine impurities. 
Comparing Figures $3 \mathrm{c}$ and $4 \mathrm{c}$, we can observe that for the sieve drum rotational speed $v_{d}=5 \mathrm{rpm}$, value of the fine impurities separation coefficient $S_{f i}$ is slightly lower than for $v_{d}=75 \mathrm{rpm}$, as the maximal $S_{f i}$ value obtained for $v_{d}=5 \mathrm{rpm}$ was $S_{f i}=0.946\left(\right.$ for $\left.\alpha=-2.5^{\circ}\right)$, whereas for $v_{d}=75 \mathrm{rpm}$ the highest $S_{f i}$ value was $S_{f i}=1.08$. The relationship between the chaff separation coefficient $S_{c h}$ and the sieve drum inclination angle is presented in Figure $4 \mathrm{~d}$. As we can see, value of the chaff separation coefficient $S_{c h}$ increases with the increase in the sieve drum inclination angle. This effect is caused by the increased amount of grains penetrating into the chaff mass.

Comparing graphs of the chaff separation coefficient $S_{c h}$ for the sieve drum rotational speed $v_{d}=5 \mathrm{rpm}$ (Figure 3d) and for $v_{d}=75 \mathrm{rpm}$ (Figure $4 \mathrm{~d}$ ) we can observe that they are reversed, one with respect to another. In the first case, $S_{c h}$ coefficient value increases with the increase of sieve drum inclination angle. In the second case, this coefficient decreases with the increase of inclination angle.

The relationship of the total coefficient of cleaning effectiveness $E$ on the sieve drum inclination angle is shown in Figure 4e. The value of the total coefficient of cleaning effectiveness $E$ has a local maximum $(\mathrm{E}=0.93)$ for inclination angle $\alpha=-1.25^{\circ}$ and decreases with the increase in the sieve drum inclination angle. This effect is caused by the reduction of sifting time and, as a result, more grains were penetrating into the chaff fraction, increasing the amount of waste. The relationship of the plump grain cleaning efficiency $q_{p g}$ on the sieve drum inclination angle is illustrated in Figure $4 \mathrm{f}$. The plump grain cleaning efficiency increased with the increase in the inclination angle. However, as the cleaning effectiveness increased, the cleaning efficiency decreased. Additionally, as can be concluded from the graphs shown in Figures $3 \mathrm{f}$ and $4 \mathrm{f}$, the plump grain cleaning efficiency for the sieve drum rotational speed value $v_{d}=5 \mathrm{rpm}$ was lower, and the maximal $q_{p g}$ value obtained was $q_{p g}=0.22 \mathrm{~kg} / \mathrm{s}$, whereas for $v_{d}=75 \mathrm{rpm}$ the maximal obtained $q_{p g}$ value was $q_{p g}=0.259 \mathrm{~kg} / \mathrm{s}$.

In summary, considering the obtained results of analysis on the influence of the sieve drum inclination angle on the course of the oat grains cleaning process, we would like to underline that the cleaning device performed its role properly within the investigated sieve drum inclination angle range. However, assuming that the most important criterion is grain purity, such cleaning device should work in the range of sieve drum inclination angle from $-2.5^{\circ}$ to $1^{\circ}$. Assuming such a value of the sieve drum inclination angle is a good trade-off between the satisfactory cleaning effectiveness and its efficiency.

For the sieve drum rotational speed $v_{d}=75 \mathrm{rpm}$, a one-way analysis of variance was performed (Table 6; at $p=0.05$ ). The conducted tests showed that for this value of the sieve drum rotational speed the sieve drum inclination angle had a significant effect on the calculated parameters. Post hoc analysis showed that obtained parameters differed significantly (Table 7). The significance level was above 0.05 for groups 2-3 (for $S_{p g}$ ), for groups 1-2, 3-4, and 4-5 (for $S_{c h}$ ), and 1-3 (for E).

Table 6. Significance level for the ANOVA analysis.

\begin{tabular}{ccccccc}
\hline$v_{\boldsymbol{d}}(\mathbf{r p m})$ & $S_{p g}$ & $S_{f g}$ & $S_{f i}$ & $S_{c h}$ & $E$ & $q_{p g}$ \\
\hline 75 & 0.00 & 0.00 & 0.00 & 0.00 & 0.00 & 0.00 \\
\hline
\end{tabular}

Table 7. Tukey's HSD test for the ANOVA.

\begin{tabular}{ccccccc}
\hline$v_{\boldsymbol{d}}(\mathbf{r p m})$ & $S_{p g}$ & $S_{f g}$ & $S_{f i}$ & $S_{c h}$ & $E$ & $\boldsymbol{q}_{p g}$ \\
\hline $1-2$ & 0.000 & 0.000 & 0.0170 .000 & $\mathbf{0 . 0 8 9}$ & 0.000 & 0.002 \\
\hline $1-3$ & 0.000 & 0.000 & 0.000 & 0.000 & $\mathbf{0 . 9 7 3}$ & 0.000 \\
\hline $1-4$ & 0.0010 .000 & 0.000 & 0.000 & 0.000 & 0.000 & 0.000 \\
\hline $1-5$ & 0.000 & 0.000 & 0.000 & 0.000 & 0.000 & 0.000 \\
\hline $2-3$ & $\mathbf{0 . 3 4 8}$ & 0.000 & 0.003 & 0.087 & 0.000 & 0.000 \\
\hline $2-4$ & 0.000 & 0.000 & 0.000 & 0.002 & 0.000 & 0.000 \\
\hline
\end{tabular}


Table 7. Cont.

\begin{tabular}{ccccccc}
\hline$v_{d}(\mathbf{r p m})$ & $S_{p g}$ & $S_{f g}$ & $S_{f i}$ & $S_{c h}$ & $E$ & $q_{p g}$ \\
\hline $2-5$ & 0.000 & 0.000 & 0.000 & 0.000 & 0.000 & 0.000 \\
\hline $3-4$ & 0.000 & 0.000 & 0.002 & $\mathbf{0 . 1 8 4}$ & 0.000 & 0.000 \\
\hline $3-5$ & 0.000 & 0.000 & 0.000 & 0.003 & 0.000 & 0.000 \\
\hline $4-5$ & 0.000 & 0.000 & 0.007 & $\mathbf{0 . 4 9 3}$ & 0.000 & 0.000 \\
\hline
\end{tabular}

1-2 indicates the significance level between group 1 and group 2, 1-3 indicates the significance level between group 1 and group 3, etc. For group $1 \alpha=-2.5^{\circ}$, for group $2 \alpha=-1.25^{\circ}$, for group $3 \alpha=0^{\circ}$, for group $4 \alpha=1.25^{\circ}$, for group $5 \alpha=2.5^{\circ}$. Means that lack significant differences are shown in bold.

\section{Discussion}

The constructed cleaning device requires research that will allow selection of technological parameters and will give information that will enable the improvement of this device.

The possibility of change of inclination angle in the range from $-7.5^{\circ}$ to $10.5^{\circ}$ (established in the cleaning device design) can be limited to the range from $-2.5^{\circ}$ to $2.5^{\circ}$. The conducted test confirmed it because cleaning efficiency decreased significantly on the borders of this range. It was confirmed by the analysis of the influence of the sieve drum inclination angle on the course of the cleaning process of oat grains, presented in this paper.

The analysis of the sieve drum inclination angle had to take into account the whole range of the rotational speed, as well as all quality indicators, such as the separation coefficients for each fraction, the total coefficient of cleaning effectiveness, and the cleaning efficiency. As this research is very labor-intensive, only two values of the sieve drum rotational speed values were analyzed. After completing investigations for these two extreme values of the sieve drum rotational speed and for five sieve drum inclination angle values, the obtained results suggest the need to continue research to determine the device settings that ensure the correct operation of the machine when separating oat grains from the mix for different atmospheric conditions and material moisture.

For this inclination angle value (from $-2.5^{\circ}$ to $2.5^{\circ}$ ) the max plump grain separation coefficient $S_{p g}$ was obtained as $S_{p g}=1.384$ for the sieve drum rotational speed $v_{d}=5 \mathrm{rpm}$ and as $S_{p g}=1.12$ for $v_{d}=75 \mathrm{rpm}$. The coefficient exceeding unity indicated that fine grains penetrated into the plump grain fraction.

The maximum value of the total cleaning efficiency coefficient $(E=0.93)$ for plump grain was observed for the sieve drum inclination angle $\alpha=-1.25^{\circ}$ for the rotational speed of the sieve drum $v_{d}=75 \mathrm{rpm}$, while for $v_{d}=5 \mathrm{rpm}$ it is $E=0.615$ for $\alpha=-2.5^{\circ}$. The effectiveness of plump grain cleaning $q_{p g}$ was highest for inclination angle $\alpha=2.5$. However, it should be noted that for this angle the total cleaning efficiency coefficient is the lowest. A high value of total coefficient of cleaning efficiency was the decisive factor to determine sieve drum inclination angle for cleaning of oat grains.

It should be emphasized that for the investigated rotary cleaning device the cleaning efficiency is closely related to the cleaning effectiveness, and the tests also confirmed this. The analysis of results of the separation and sifting of oat grains from impurities showed that for the rotational speed $v_{d}=5 \mathrm{rpm}$, the value of the total cleaning efficiency coefficient has lower values ( $E=0.565$ to 0.605$)$ for the entire investigated range of sieve drum inclination angle. Whereas, for the sieve drum rotational speed $v_{d}=75 \mathrm{rpm}$ the value of the total leaning efficiency coefficient has bigger values ( $E=0.86$ to 0.93 ) for the entire investigated range of sieve drum inclination angle. The improvement in grain cleaning efficiency was caused by increases in the centrifugal force facilitating sifting. The optimal value of sieve drum inclination angle is $\alpha=-1.25^{\circ}$ for $v_{d}=75 \mathrm{rpm}$ (all indicators except $q_{p g}$ ). For the sieve drum rotational speed $v_{d}=5 \mathrm{rpm}$ only fine impurities separation coefficient $S_{f i}$ has the optimum for the sieve drum inclination angle $\alpha=-1.25^{\circ}$.

We are planning to continue the investigations of the rotary cleaning device, using the presented results as guidelines, in order to determine the optimal settings of the device for its proper operation 
and to obtain the highest available effectiveness and efficiency. Controlling the process in which the material is organic is difficult because these materials change their properties over time. Such processes are usually nonstationary and nonlinear, for such processes advanced control algorithms must be used [38].

The constructed cleaning device can be useful in small and medium farms where ecological production is often carried out as part of sustainable agriculture. The constructed device can be powered by electricity obtained from natural sources (sun, air, water, etc.). The device is designed for the separation and cleaning of various agricultural products, not only cereal grains but also peas, beans, broad beans, and other products.

\section{Conclusions}

The tested rotary cleaning device adapted for cleaning oat grains, turned out to be the correct construction that fulfills its role well. The pre-applied inclination angle of the sieve drum $\left(\alpha=0^{\circ}\right)$ as a natural basic position of the sieve drum was properly selected. The optimal value of most coefficient is for the angle $\alpha=-1.25^{\circ}$. However, the effectiveness of plump grain cleaning $\mathrm{q}_{\mathrm{pg}}$ suggests increasing the value of this angle (for example $\alpha=0^{\circ}$ ).

The angle of device location has a significant impact on the quality of the cleaning and grain separation process, which was confirmed by statistical analyses.

Author Contributions: Conceptualization, Z.K. (Zbigniew Krzysiak); methodology, Z.K. (Zbigniew Krzysiak), J.Z. and Z.K. (Zdzisław Kaliniewicz); software, W.S. and M.B.; validation, D.P. and M.B.; formal analysis, Z.K. and W.S.; investigation, Z.K. (Zbigniew Krzysiak) and J.Z.; data curation, Z.K. (Zbigniew Krzysiak) and W.S.; writing - original draft preparation, Z.K. (Zbigniew Krzysiak) and W.S.; writing—review and editing, W.S. All authors have read and agreed to the published version of the manuscript.

Funding: This research received no external funding.

Conflicts of Interest: The authors declare no conflict of interest.

\section{References}

1. Learn Europe-Educational Resources about the European Union. Available online: https://www.learneurope. eu/files/2513/7525/9029/Resumen_de_la_PAC_en.pdf (accessed on 29 January 2020).

2. Union, P.O. of the E. Agriculture: A Partnership between Europe and Farmers. Available online: https://op.europa.eu:443/en/publication-detail/-/publication/f08f5f20-ef62-11e6-8a35-01aa75ed71a1/ language-en/format-PDF (accessed on 14 January 2020).

3. Van Huy, L.; Chi, M.T.T.; Lobo, A.; Nguyen, N.; Long, P.H. Effective Segmentation of Organic Food Consumers in Vietnam Using Food-Related Lifestyles. Sustain. Switz. 2019, 11, 11051237. [CrossRef]

4. Nagy-Pércsi, K.; Fogarassy, C. Important Influencing and Decision Factors in Organic Food Purchasing in Hungary. Sustain. Switz. 2019, 11, 11216075. [CrossRef]

5. de Olde, E.; Oudshoorn, F.; Bokkers, E.; Stubsgaard, A.; Sørensen, C.; de Boer, I. Assessing the Sustainability Performance of Organic Farms in Denmark. Sustainability 2016, 8, 957. [CrossRef]

6. Zrównoważone rolnictwo w Polsce. Available online: www.Nowoczesnerolnictwo.Info/RolnictwoZrownowazone/Zrownowazone-Rolnictwo-w-Polsce (accessed on 14 January 2020).

7. Jokić, G.; Prole, S.; Butaš, D.; Sedlar, A.; Bugarin, R.; Turan, J. Analysis the Parameters of Quality Sunflower Hybrid Seed after Processing on Fine Cleaner, Trijer Machine and Gravity Separator. Savrem. Poljopr. Teh. 2016, 42, 25-38. [CrossRef]

8. Uhlarik, A.; Popov, S.; Karagić, Đ.; Ponjičan, O.; Turan, J. Alfalfa Seed Cleaning Using a Magnetic Separator. J. Process. Energy Agric. 2018, 22, 192-195. [CrossRef]

9. Serna-Saldivar, S. Cereal Grains: Properties, Processing, and Nutritional Attributes. Available online: https://www.crcpress.com/Cereal-Grains-Properties-Processing-and-Nutritional-Attributes/SernaSaldivar/p/book/9781439815601 (accessed on 2 March 2020).

10. Grochowicz, J. Machines for Cleaning and Sorting; Agricultural University in Lublin: Lublin, Poland, 1994. (In Polish) 
11. Dursun, I.; Tuğrul, K.M.; Dursun, E. Some Physical Properties of Sugarbeet Seed. J. Stored Prod. Res. 2007, 43, 149-155. [CrossRef]

12. Adewumi, B.; Ogunlowo, A.; Ademosun, O. Preliminary Investigation on the Distribution and Spread Pattern of Cowpea in a Cross Flow Grain Separator. GIGR J. 2006, 8, 1-12.

13. Stoleru, V.; Munteanu, N.; Istrate, A. Perception towards Organic vs. Conventional Products in Romania. Sustain. Switz. 2019, 11. [CrossRef]

14. Panasiewicz, M.; Sobczak, P.; Mazur, J.; Zawiślak, K.; Andrejko, D. The Technique and Analysis of the Process of Separation and Cleaning Grain Materials. J. Food Eng. 2012, 109, 603-608. [CrossRef]

15. Reichert, R.D. Air Classification of Peas (Pisum Sativum) Varying Widely in Protein Content. J. Food Sci. 1982, 47, 1263-1267. [CrossRef]

16. Krzysiak, Z.; Samociuk, W.; Skic, A.; Bartnik, G.; Zarajczyk, J.; Szmigielski, M.; Dziki, D.; Wierzbicki, S.; Krzywonos, L. Effect of Sieve Drum Inclination Angle on Wheat Grain Cleaning in a Novel Rotary Cleaning Device. Trans. ASABE 2017, 60, 1751-1758. [CrossRef]

17. Krzysiak, Z. Cleaning Device for Seeds. Patent PL65892 (Y1), 30 April 2012. (In Polish)

18. Kaliniewicz, Z. Influence of Cereal Seed Orientation on External Friction Coefficients. Trans. ASABE 2016, 59, 1073-1081. [CrossRef]

19. Neale, M.A.; Hobson, R.N.; Price, J.S.; Bruce, D.M. Effectiveness of Three Types of Grain Separator for Crop Matter Harvested with a Stripping Header. Biosyst. Eng. 2003, 84, 177-191. [CrossRef]

20. Ozturk, T.; Kara, M.; Elkoca, E.; Ercisli, S. Physico-Chemical Grain Properties of New Common Bean Cv. "Elkoca-05." Sci. Res. Essays 2008, 4, 088-093.

21. Ozturk, T.; Esen, B. Physical and Mechanical Properties of Barley. Agric. Trop. Subtrop. 2008, 41, 117-121.

22. Rajabipour, A. Effect of Moisture on Terminal Velocity of Wheat Varieties. Int. J. Agric. Biol. 2006, 8, 10-13.

23. Roz, M.; Rinaudo, C.; Rossanigo, P.; Polati, S.; Gennaro, M.C. Gravitational Grain Size Separation of Contaminated Ground: Potential and Limits. Appl. Clay Sci. 2006, 31, 85-89. [CrossRef]

24. Simonyan, J.K. Investigating Grain Separation and Cleaning Efficiency Distribution of a Conventional Stationary Rasp-Bar Sorghum Thresher. Agric. Eng. Int. CIGR J. 2008, 10, 1-12.

25. Kutz, M. Handbook of Farm Dairy and Food Machinery; Elsevier Science: London, UK, 2019.

26. Kaliniewicz, Z.; Zuk, Z.; Krzysiak, Z. Influence of Steel Plate Roughness on the Frictional Properties of Cereal Kernels. Sustainability 2018, 10, 1003. [CrossRef]

27. Gibiński, M.; Gumul, D.; Korus, J. Health-Promoting Properties of Oats and Oat Products. Żywność Nauka Technol. Jakość 2005, 12, 49-60. (In Polish)

28. Kiryluk, J. Oat Bran-A Product That Wins the World. Przegl. Zboż. Młyn 2004, 48, 12-14. (In Polish)

29. Decker, E.A.; Rose, D.J.; Stewart, D. Processing of Oats and the Impact of Processing Operations on Nutrition and Health Benefits. Br. J. Nutr. 2014, 112, S58-S64. [CrossRef]

30. Stevenson, D.G.; Inglett, G.E. Oat Dietary Fiber: Commercial Processes and Functional Attributes. In Oats: Chemistry and Technology, 2nd ed.; AACC International, Inc.: St. Paul, MN, USA, 2011; pp. 321-331.

31. Hebda, T.M. Geometric Features of Grains of Selected Cereal Varieties. Agric. Eng. 2007, 5, 187-193.

32. Al-Mahasneh, M.A.; Rababah, T.M. Effect of Moisture Content on Some Physical Properties of Green Wheat. J. Food Eng. 2007, 79, 1467-1473. [CrossRef]

33. Kalkan, F.; Kara, M. Handling, Frictional and Technological Properties of Wheat as Affected by Moisture Content and Cultivar. Powder Technol. 2011, 213, 116-122. [CrossRef]

34. Rababah, T.M.; Ereifej, K.I.; Al-Mahasneh, M.A.; Alhamad, M.N.; Alrababah, M.A.; Muhammad, A.H. The Physicochemical Composition of Acorns for Two Mediterranean Quercus Species. Jordan J. Agric. Sci. 2008, 4, 131-137.

35. Krzysiak, Z. Construction of an Innovative Rotary Grain Cleaning Device. Mechanik 2012, 2, 153. (In Polish)

36. Samociuk, W.; Wyciszkiewicz, A.; Gołacki, K.; Otto, T. Ryzyko Wystqpienia Powaznej Awarii Reaktora Syntezy Mocznika. Przemysl Chem. 2017, 96, 1763-1766. [CrossRef]

37. Formela, K.; Bogucki, M. Use of Response Surface Methodology in Characterization of Properties of Recycled High Density Polyethylene/Ground Tire Rubber Compositions. Polimery/Polymers 2014, 59, 488-494. [CrossRef]

38. Wolszczak, P.; Samociuk, W. Identification of Non-Stationary and Non-Linear Drying Processes. In Topics in Nonlinear Mechanics and Physics; Springer: Singapore, 2019; Volume 228, pp. 295-309. [CrossRef] 
39. Hamed, Y.S.; Aly, A.A.; Saleh, B.; Alogla, A.F.; Aljuaid, A.M.; Alharthi, M.M. Nonlinear Structural Control Analysis of an Offshore Wind Turbine Tower System. Processes 2020, 8, 22. [CrossRef]

40. Krzysiak, Z. Evaluation of Rye Grain Cleaning in a New, Rotary Cleaning Device. Mechanik 2013, $2,143$. (In Polish)

41. Krzysiak, Z. Selection of the Range of Operating Parameters of the New Rotary Cleaning Device. Mechanik 2013, 7, 595. (In Polish) 